\title{
Minimum-Energy Bivariate Wavelet Frame with Arbitrary Dilation Matrix
}

\author{
Fengjuan Zhu, Qiufu Li, and Yongdong Huang \\ School of Mathematics and Information Science, Beifang University of Nationalities, Yinchuan 750021, China \\ Correspondence should be addressed to Yongdong Huang; nxhyd74@126.com
}

Received 31 January 2013; Revised 10 June 2013; Accepted 18 June 2013

Academic Editor: Li Weili

Copyright (C) 2013 Fengjuan Zhu et al. This is an open access article distributed under the Creative Commons Attribution License, which permits unrestricted use, distribution, and reproduction in any medium, provided the original work is properly cited.

In order to characterize the bivariate signals, minimum-energy bivariate wavelet frames with arbitrary dilation matrix are studied, which are based on superiority of the minimum-energy frame and the significant properties of bivariate wavelet. Firstly, the concept of minimum-energy bivariate wavelet frame is defined, and its equivalent characterizations and a necessary condition are presented. Secondly, based on polyphase form of symbol functions of scaling function and wavelet function, two sufficient conditions and an explicit constructed method are given. Finally, the decomposition algorithm, reconstruction algorithm, and numerical examples are designed.

\section{Introduction}

Frames theory is one of the efficient tools in the signal processing. It was introduced by Duffin and Schaeffer [1] and was used to deal with problems in nonharmonic Fourier series. However, people did not pay enough attention to frames theory for a long time. When wavelets theory was booming, Daubechies et al. [2] defined the affine frame (wavelet frame) by combining the theory of continuous wavelet transform with frame. After that, people started to research frames and its application again. Benedetto and $\mathrm{Li}$ [3] gave the definition of frame multiresolution analysis (FMRA), and their work laid the foundation for other people to do further research. Frames not only can overcome the disadvantages of wavelets and multivariate wavelets but also increase redundancy, and the numerical computation becomes much more stable using frames to reconstruct signal. With well timefrequency localization and shift invariance, frames can be designed more easily than wavelets or multivariate wavelets. At present, frames theory has been widely used in theoretical and applicable domains [4-18], such as signal analysis, image processing, numerical calculation, Banach space theory, and Besov space theory.

In 2000, Chui and $\mathrm{He}$ [5] proposed the concept of minimum-energy wavelet frames. The minimum-energy wavelet frames reduce the computational, and maintain the numerical stability, and do not need to search dual frames in the decomposition and reconstruction of functions (or signals). Therefore, many people paid more attention to the study of minimum-energy wavelet frames. Petukhov [6] studied the (minimum-energy) wavelet frames with symmetry. Huang and Cheng [7] studied the construction and characterizations of the minimum-energy wavelet frames with arbitrary integer dilation factor. Gao and Cao [8] researched the structure of the minimum-energy wavelet frames on the interval $[0,1]$ and its application on signal denoising. Liang and Zhao [9] studied the minimum-energy multiwavelet frames with dilation factor 2 and multiplicity 2 and gave a characterization and a necessary condition of minimum-energy multiwavelets frames. Huang et al. $[10,11]$ studied minimum-energy multiwavelet frames and wavelet frames on the interval $[0,1]$ with arbitrary dilation factor. It was well known that a majority of real-world signals are multidimensional, such as graphic and video signal. For this reason, many people studied multivariate wavelets and multivariate wavelet frames [12-18]. In this paper, in order to deal with multidimensional signals and combine organically the minimum-energy wavelet frames with the significant properties of multivariate wavelets, minimum-energy bivariate wavelet frames with arbitrary dilation matrix are studied. 
The organization of this paper is as follows. In Section 2, we give preliminaries and basic definitions. Then, in Section 3, the main results are described. In Section 4, we present the decomposition and reconstruction formulas of minimum-energy bivariate wavelet frames. Finally, numerical examples are given in Section 5.

\section{Preliminaries and Basic Definitions}

2.1. Basic Concept and Notation. Let us recall the concept of dilation matrix and give some notations.

Definition 1 (see [18]). Let $A$ be the $2 \times 2$ integer matrix. Suppose that its eigenvalues have a modulus strictly greater than 1 , then $A$ is called a dilation matrix.

(1) Throughout this paper, let $\mathbb{Z}, \mathbb{R}$ denote the set of integers and real numbers, respectively. $\mathbb{Z}^{2}, \mathbb{R}^{2}$ denote the set of 2-tuple integers and two-dimensional Euclidean space, respectively. For a given dilation matrix $A$, let $\left\{\mu_{i}, i=0,1, \ldots, s-1\right\}$ be a complete set of representatives of $\mathbb{Z}^{2} / A \mathbb{Z}^{2}$, where $s=|\operatorname{det}(A)|$.

(2) Let $L^{2}\left(\mathbb{R}^{2}\right)=\left\{f: \int_{\mathbb{R}^{2}}|f(x)|^{2} d x<\infty\right\}$ and $l^{2}\left(\mathbb{Z}^{2}\right)=$ $\left\{s: \sum_{m \in \mathbb{Z}^{2}}\left|s_{m}\right|^{2} d x<\infty\right\}$. For any $f, g \in L^{2}\left(\mathbb{R}^{2}\right)$, the inner product, norm, and the Fourier transform are defined, respectively, by

$$
\begin{gathered}
\langle f(x), g(x)\rangle=\int_{\mathbb{R}^{2}} f(x) \overline{g(x)} d x, \quad\|f\|^{2}=\langle f, f\rangle, \\
\widehat{f}(\xi)=\frac{1}{\sqrt{2 \pi}} \int_{\mathbb{R}^{2}} f(x) e^{-i x \cdot \xi} d x .
\end{gathered}
$$

(3) For any function $f \in L^{2}\left(\mathbb{R}^{2}\right), f_{j, k}(x)$ is defined by

$$
f_{j, k}(x)=s^{j / 2} f\left(A^{j} x-k\right), \quad j \in \mathbb{Z}, k \in \mathbb{Z}^{2} .
$$

(4) We sort the elements of $\mathbb{Z}^{2}$ by lexicographical order. That is, for any $k=\left(k_{1}, k_{2}\right)^{T}, l=\left(l_{1}, l_{2}\right)^{T}, k, l \in \mathbb{Z}^{2}$, and let

$$
m=\left(k_{1}-l_{1}, k_{2}-l_{2}\right)
$$

then $k=l$ denotes that every component of $m$ is zero, $k>$ $l$ denotes that the first nonzero component of $m$ is positive, and $k<l$ denotes that the first nonzero component of $m$ is negative.

(5) For any $z=\left(z_{1}, z_{2}\right)^{T}, z_{1} \neq 0, z_{2} \neq 0, k=\left(k_{1}, k_{2}\right)^{T} \in$ $\mathbb{R}^{2}, z^{-1}, z^{k}$ are defined, respectively, by

$$
z^{-1}=\left(z_{1}^{-1}, z_{2}^{-1}\right)^{T}, \quad z^{k}=\prod_{i=1}^{2} z_{i}^{k_{i}} .
$$

(6) For any $A \in \mathbb{R}^{2 \times 2}, z^{A}$ is defined by

$$
z^{A}=\left(z^{A_{1}}, z^{A_{2}}\right)^{T}
$$

where $A_{i}$ is the $i$-th column of $A$.

Now, we give the definition of frame.
Definition 2 (see [18]). Let $\mathbb{M}$ be a complex and separable Hilbert space. A sequence $\left\{f_{k}: k \in \mathbb{Z}\right\}$ is a frame for $\mathbb{H}$ if there exist constants $0<A, B<\infty$, such that, for any $f \in \mathbb{H}$,

$$
A\|f\|^{2} \leq \sum_{k \in \mathbb{Z}}\left|\left\langle f, f_{k}\right\rangle\right|^{2} \leq B\|f\|^{2} .
$$

The numbers $A, B$ are called frame bounds.

A frame is tight if we can choose $A=B$ as frame bounds in the Definition 2. If a frame ceases to be a frame when an arbitrary element is removed, it is called an exact frame. When $A=B=1$, then

$$
f=\sum_{k \in \mathbb{Z}}\left\langle f, f_{k}\right\rangle f_{k}, \quad \forall f \in \mathbb{H} .
$$

\subsection{Minimum-Energy Bivariate Wavelet Frame}

Definition 3 (see [18]). A bivariate frame multiresolution analysis (FMRA) for $L^{2}\left(\mathbb{R}^{2}\right)$ consists of a sequence of closed subspaces $\left\{V_{j}\right\}_{j \in \mathbb{Z}}$ and a function $\phi \in V_{0}$ such that

(1) $V_{j} \subset V_{j+1}, j \in \mathbb{Z}$

(2) $\bigcap_{j \in \mathbb{Z}} V_{j}=\{0\}, \overline{\bigcup_{j \in \mathbb{Z}} V_{j}}=L^{2}\left(\mathbb{R}^{2}\right)$;

(3) $f(x) \in V_{j} \Leftrightarrow f(A x) \in V_{j+1}, j \in \mathbb{Z}, x \in \mathbb{R}^{2}$;

(4) $\{\phi(x-n)\}_{n \in \mathbb{Z}^{2}}$ is a frame for $V_{0}$,

where function $\phi(x)$ is called scaling function of FMRA.

Since $V_{0} \subset V_{1}, \phi(x)$ satisfies two-scale equation (refinable equation)

$$
\phi(x)=\sum_{k \in \mathbb{Z}^{2}} p_{k} \phi(A x-k),
$$

where $\left\{p_{k}\right\}_{k \in \mathbb{Z}^{2}} \in l^{2}\left(\mathbb{Z}^{2}\right)$. The Fourier transform of (8) is

$$
\widehat{\phi}(\xi)=m_{0}\left(A^{-T} \xi\right) \widehat{\phi}\left(A^{-T} \xi\right), \quad \xi \in \mathbb{R}^{2},
$$

where

$$
m_{0}(\xi)=\frac{1}{|\operatorname{det}(A)|} \sum_{k \in \mathbb{Z}^{2}} p_{k} e^{-i \xi^{T} k}=\frac{1}{s} \sum_{k \in \mathbb{Z}^{2}} p_{k} z^{k}
$$

where $m_{0}(\xi)$ is the symbol function of scaling function $\phi(x)$, $z=e^{-i \xi^{T}}$.

For simplicity, in this paper, we suppose that any symbol function is trigonometric polynomial, and scaling function and wavelet function are compactly supported.

Definition 4. Let $\phi \in L^{2}\left(\mathbb{R}^{2}\right)$ satisfies $\widehat{\phi} \in L^{\infty}$ and $\widehat{\phi}$ continuous at 0 and $\widehat{\phi}(0)=1$. Suppose that $\phi$ generates a sequence of nested closed subspaces $\left\{V_{j}\right\}_{j \in \mathbb{Z}}$, then $\Psi=$ $\left\{\psi^{1}, \psi^{2}, \ldots, \psi^{N}\right\} \subset V_{1}$ is called a minimum-energy bivariate wavelet frame associated with $\phi$ if for all $f \in L^{2}\left(\mathbb{R}^{2}\right)$ :

$$
\sum_{k \in \mathbb{Z}^{2}}\left|\left\langle f, \phi_{1, k}\right\rangle\right|^{2}=\sum_{k \in \mathbb{Z}^{2}}\left|\left\langle f, \phi_{0, k}\right\rangle\right|^{2}+\sum_{l=1}^{N} \sum_{k \in \mathbb{Z}^{2}}\left|\left\langle f, \psi_{0, k}^{l}\right\rangle\right|^{2} .
$$


By the Parseval identity, minimum-energy bivariate wavelet frame $\Psi$ must be a tight frame for $L^{2}\left(\mathbb{R}^{2}\right)$ with frame bound being equal to 1 . At the same time, the formula (11) is equivalent to

$$
\begin{aligned}
\sum_{k \in \mathbb{Z}^{2}}\left\langle f, \phi_{1, k}\right\rangle \phi_{1, k}= & \sum_{k \in \mathbb{Z}^{2}}\left\langle f, \phi_{0, k}\right\rangle \phi_{0, k} \\
& +\sum_{l=1}^{N} \sum_{k \in \mathbb{Z}^{2}}\left\langle f, \psi_{0, k}^{l}\right\rangle \psi_{0, k}^{l}, \quad \forall f \in L^{2}\left(\mathbb{R}^{2}\right) .
\end{aligned}
$$

The interpretation of minimum-energy bivariate wavelet frame will be clarified later.

Consider $\Psi=\left\{\psi^{1}, \psi^{2}, \ldots, \psi^{N}\right\} \subset V_{1}$, with

$$
\psi^{l}(x)=\sum_{k \in \mathbb{Z}^{2}} q_{k}^{l} \phi(A x-k), \quad l=1,2, \ldots, N .
$$

Using Fourier transform on the previous equation, we can get their symbols as follows:

$$
m_{l}(\xi)=\frac{1}{s} \sum_{k \in \mathbb{Z}^{2}} q_{k}^{l} e^{-i \xi^{T} k}=\frac{1}{s} \sum_{k \in \mathbb{Z}^{2}} q_{k}^{l} z^{k}, \quad l=1,2, \ldots, N,
$$

where $z=e^{-i \xi^{T}}$.

With $m_{0}(\xi), m_{1}(\xi), \ldots, m_{N}(\xi)$, we formulate the $s \times(N+1)$ matrix $M(\xi)$ :

$M(\xi)$

$$
=\left(\begin{array}{cccc}
m_{0}(\xi) & m_{1}(\xi) & \cdots & m_{N}(\xi) \\
m_{0}\left(\xi+2 A^{-T} \mu_{1} \pi\right) & m_{1}\left(\xi+2 A^{-T} \mu_{1} \pi\right) & \cdots & m_{N}\left(\xi+2 A^{-T} \mu_{1} \pi\right) \\
\vdots & \vdots & & \vdots \\
m_{0}\left(\xi+2 A^{-T} \mu_{s-1} \pi\right) & m_{1}\left(\xi+2 A^{-T} \mu_{s-1} \pi\right) & \cdots & m_{N}\left(\xi+2 A^{-T} \mu_{s-1} \pi\right)
\end{array}\right),
$$

and $M^{*}(\xi)$ denotes the complex conjugate of the transpose of $M(\xi)$.

\section{Main Result}

In this section, we give a complete characterization of minimum-energy bivariate wavelet frames with arbitrary dilation matrix and two sufficient conditions and a necessary condition of minimum-energy bivariate wavelet frame associated with the given scaling function.

Proposition 5. Suppose that $A$ is a dilation matrix; let

$$
\mathscr{A}=\left(\begin{array}{cccc}
e^{i 2 \pi \mu_{1}^{T} A^{-1} \mu_{1}} & e^{i 2 \pi \mu_{1}^{T} A^{-1} \mu_{2}} & \cdots & e^{i 2 \pi \mu_{1}^{T} A^{-1} \mu_{s-1}} \\
e^{i 2 \pi \mu_{2}^{T} A^{-1} \mu_{1}} & e^{i 2 \pi \mu_{2}^{T} A^{-1} \mu_{2}} & \cdots & e^{i 2 \pi \mu_{2}^{T} A^{-1} \mu_{s-1}} \\
\vdots & \vdots & & \vdots \\
e^{i 2 \pi \mu_{s-1}^{T} A^{-1} \mu_{1}} & e^{i 2 \pi \mu_{s-1}^{T} A^{-1} \mu_{2}} & \cdots & e^{i 2 \pi \mu_{s-1}^{T} A^{-1} \mu_{s-1}}
\end{array}\right) ;
$$

then, $\mathscr{A}$ is invertible matrix.

The following theorem presents the equivalent characterizations of the minimum-energy bivariate wavelet frames with arbitrary dilation matrix.
Theorem 6. Suppose that the symbols $m_{l}(\xi), l=0,1, \ldots, N$ in (10) and (14) are Laurent polynomial, and generate the refinable function $\phi(x)$ and $\Psi=\left\{\psi^{1}, \psi^{2}, \ldots, \psi^{N}\right\}$. If $\hat{\phi}$ is continuous at 0 and $\widehat{\phi}(0)=1$ and $\phi(x)$ generates a nested closed subspaces sequence $\left\{V_{j}\right\}_{j \in \mathbf{Z}}$, then the following statements are equivalent.

(1) $\Psi$ is a minimum-energy bivariate wavelet frame with arbitrary dilation matrix $A$ associated with $\phi(x)$.

(2)

$$
M(\xi) M^{*}(\xi)=I_{s}, \quad \forall \xi \in \mathbb{R}^{2} .
$$

(3) $\quad \alpha_{m l}=\sum_{k \in \mathbb{Z}^{2}}\left(p_{m-A k}^{*} p_{l-A k}+\sum_{i=1}^{N} q_{m-A k}^{i *} q_{l-A k}^{i}\right)$

$$
-s \delta_{m l}=0, \quad \forall m, l \in \mathbb{Z}^{2} .
$$

Proof. By using the two-scale relations (8) and (13) and notation $\alpha_{m l}$, then formula (12) is equivalent to

$$
\sum_{m, l \in \mathbb{Z}^{2}} \alpha_{m l}\langle f, \phi(A x-m)\rangle \phi(A x-l)=0, \quad \forall f \in L^{2}\left(\mathbb{R}^{2}\right) .
$$

On the other hand, formula (17) can be reformulated as

$$
\sum_{l=0}^{N}\left|m_{l}(\xi)\right|^{2}=1
$$

$$
\sum_{l=0}^{N} m_{l}(\xi) m_{l}^{*}\left(\xi+2 A^{-1} \mu_{j} \pi\right)=0, \quad j=1, \ldots, s-1
$$

which is equivalent to

$$
\begin{gathered}
\sum_{l=0}^{N} m_{l}(\xi) \sum_{j=0}^{s-1} m_{l}^{*}\left(\xi+2 A^{-1} \mu_{j} \pi\right)=1, \\
\sum_{l=0}^{N} m_{l}(\xi)\left(m_{0}^{*}(\xi)-\sum_{j=1}^{s-1} m_{l}^{*}\left(\xi+2 A^{-1} \mu_{j} \pi\right)\right)=1, \\
\sum_{l=0}^{N} m_{l}(\xi)\left(\sum_{j=0}^{s-1} m_{l}^{*}\left(\xi+2 A^{-1} \mu_{j} \pi\right)-2 m_{l}\left(\xi+2 A^{-1} \mu_{n} \pi\right)\right)=1, \\
n=1, \ldots, s-1 ; \xi \in \mathbb{R}^{2},
\end{gathered}
$$

or

$$
\begin{gathered}
\sum_{l=0}^{N} m_{l}(\xi) \sum_{k \in \mathbb{Z}^{2}} q_{-A k}^{l *} z^{A k}=1, \\
\sum_{l=0}^{N} m_{l}(\xi)\left(\sum_{n=1}^{s-1} \sum_{k \in \mathbb{Z}^{2}} q_{\mu_{n}}^{l *} *_{k} z^{A k-\mu_{n}}\right)=s-1, \\
\sum_{l=0}^{N} m_{l}(\xi)\left(\sum_{n=1}^{s-1} e^{i 2 \mu_{j}^{T} A^{-1} \mu_{n} \pi} \sum_{k \in \mathbb{Z}^{2}} q_{\mu_{n}-A k}^{l *} z^{A k-\mu_{n}}\right)=-1, \\
j=1, \ldots, s-1 ; \xi \in \mathbb{R}^{2},
\end{gathered}
$$


where $q_{k}^{0}=p_{k}, k \in \mathbb{Z}^{2}$. Since $\mathscr{A}$ is an invertible matrix, the previous equation is equivalent to

$$
\sum_{l=0}^{N} m_{l}(\xi) \sum_{k \in \mathbb{Z}^{2}} q_{\mu_{n}-A k}^{l *} z^{A k-\mu_{n}}=1, \quad n=0,1, \ldots, s-1 .
$$

We multiply the identities in (23) by $z^{\mu_{n}} \widehat{\phi}\left(A^{-T} \xi\right), n=$ $0,1, \ldots, s-1$, respectively, where $z=e^{-i \xi^{T} A^{-1}}$, and we get

$\widehat{\phi}\left(A^{-T} \xi\right) z^{\mu_{n}}=\sum_{l=0}^{N} \sum_{k \in \mathbb{Z}^{2}} q_{\mu_{n}-A k}^{l *} z^{A k} \widehat{\psi}^{l}(\xi), \quad n=0,1, \ldots, s-1 ;$

let $\psi^{0}=\phi$. Take the Fourier transform on the two sides of the previous formula, (23) is equivalent to

$$
s \phi\left(A x-\mu_{n}\right)=\sum_{l=0}^{N} \sum_{k \in \mathbb{Z}^{2}} q_{\mu_{n}-A k}^{l *} \psi^{l}(x-k), \quad n=0,1, \ldots, s-1 .
$$

Thus,

$$
s \phi(A x-\mu)=\sum_{l=0}^{N} \sum_{k \in \mathbb{Z}^{2}} q_{\mu-A k}^{l *} \psi^{l}(x-k), \quad \forall \mu \in \mathbb{Z}^{2} .
$$

By using the two-scale relations (8) and (13), we can rewrite formula (26) as

$$
\sum_{m \in \mathbb{Z}^{2}} \alpha_{m l} \phi(A x-m)=0, \quad \forall l \in \mathbb{Z}^{2} .
$$

In other words, the proof of Theorem 6 reduces to the proof of the equivalences of (18), (19), and (27).

It is clear that $(18) \Rightarrow(27) \Rightarrow(19)$. In order to prove $(19) \Rightarrow(18)$, let $f \in L^{2}\left(\mathbb{R}^{2}\right)$ be any compactly supported function. Let

$$
\beta_{l}(f)=\left\langle f, \sum_{m \in \mathbb{Z}^{2}} \alpha_{m l} \phi(A x-m)\right\rangle, \quad l \in \mathbb{Z}^{2} .
$$

Then by using the properties that, for every fixed $m, \alpha_{m l}=0$ except for finitely many $l$, and both $\phi$ and $f$ have compact support, it is clear that only finitely many of the values $\beta_{l}(f)$ is nonzero. Now, since $\widehat{\phi}(\xi)$ is a nontrivial function, by taking the Fourier transform of (19), it follows that the trigonometric polynomial $\sum_{l \in \mathbb{Z}^{2}} \beta_{l}(f) e^{-i \xi^{T} A^{-T} l} \equiv 0$. Obviously, $\beta_{l}(f)=0, l \in$ $\mathbb{Z}^{2}$. By choosing $f=\sum_{m \in \mathbb{Z}^{2}} \alpha_{m l} \phi(A x-m)$, we get formula (27).

By taking the Fourier transform of (27), we get $\alpha_{m l}=$ $0, m, l \in \mathbb{Z}^{2}$. The proof of Theorem 6 is completed.

Theorem 6 characterizes the necessary and sufficient condition for the existence of the minimum-energy bivariate wavelet frames associated with $\phi$. But it is not a good choice to use this theorem to construct the minimum-energy bivariate wavelet frames with arbitrary dilation matrix. For convenience, we need to present some sufficient conditions in terms of the symbol functions.
Theorem 7. A compactly supported refinable function $\phi \epsilon$ $L^{2}\left(\mathbb{R}^{2}\right)$, with $\widehat{\phi}$ continuous at 0 and $\widehat{\phi}(0)=1$, and $m_{0}(\xi)$ is the symbol function of $\phi$. Let $\Psi=\left\{\psi^{1}, \psi^{2}, \ldots, \psi^{N}\right\}$ be the minimum-energy multiwavelet frames associated with $\phi$; then

$$
\sum_{n=0}^{s-1}\left|m_{0}\left(\xi+2 A^{-1} \mu_{n} \pi\right)\right|^{2} \leq 1, \quad \forall \xi \in \mathbb{R}^{2} .
$$

Proof. Let $D(\xi)$ be the first column of $M(\xi)$ and $M(\xi)=$ $(D(\xi), Q(\xi))$. Then,

$$
D(\xi) D^{*}(\xi)+Q(\xi) Q^{*}(\xi)=I_{s} .
$$

Since $Q(\xi) Q^{*}(\xi)$ is a Hermitian matrix, the matrix $I_{s}-$ $D(\xi) D^{*}(\xi)$ is positive semidefinite.

We have

$$
\begin{gathered}
\left(\begin{array}{cc}
I_{s} & D(\xi) \\
D^{*}(\xi) & 1
\end{array}\right)\left(\begin{array}{cc}
I_{s} & -D(\xi) \\
-D^{*}(\xi) & 1
\end{array}\right) \\
=\left(\begin{array}{cc}
I_{s}-D(\xi) D^{*}(\xi) & \\
& 1-D^{*}(\xi) D(\xi)
\end{array}\right) \\
\operatorname{det}\left(\begin{array}{cc}
I_{s} & D(\xi) \\
D^{*}(\xi) & 1
\end{array}\right)=\operatorname{det}\left(\begin{array}{cc}
I_{s} & D(\xi) \\
& 1-D^{*}(\xi) D(\xi)
\end{array}\right), \\
\operatorname{det}\left(\begin{array}{cc}
I_{s} & -D(\xi) \\
-D^{*}(\xi) & 1
\end{array}\right)=\operatorname{det}\left(\begin{array}{cc}
I_{s} & -D(\xi) \\
& 1-D^{*}(\xi) D(\xi)
\end{array}\right) .
\end{gathered}
$$

Therefore,

$$
\begin{aligned}
\operatorname{det}\left(I_{s}-D(\xi) D^{*}(\xi)\right)\left(1-D^{*}(\xi) D(\xi)\right) \\
=\left(1-D^{*}(\xi) D(\xi)\right)\left(1-D^{*}(\xi) D(\xi)\right),
\end{aligned}
$$

that is,

$$
1-D^{*}(\xi) D(\xi) \geq 0 .
$$

The proof of Theorem 7 is completed.

According to the Theorem 7, there may not exist minimum-energy bivariate wavelet frame associated with a given scaling function. If there exists a minimum-energy bivariate wavelet frame, then the symbol function of scaling function must satisfy (29). Based on the polyphase forms of $m_{0}(z), m_{1}(z), \ldots, m_{N}(z)$, we give two sufficient conditions.

Let $z=e^{-i \xi^{T}}$; then

$$
\begin{aligned}
m_{0}(\xi) & =\frac{1}{s} \sum_{k \in \mathbb{Z}^{2}} p_{k} e^{-i \xi^{T} k}=\frac{1}{s} \sum_{i=0}^{s-1} \sum_{k \in \mathbb{Z}^{2}} p_{\mu_{i}+A k} e^{-i \xi^{T}\left(\mu_{i}+A k\right)} \\
& =\frac{1}{s} \sum_{i=0}^{s-1} e^{-i \xi^{T} \mu_{i}} \sum_{k \in \mathbb{Z}^{2}} p_{\mu_{i}+A k} e^{-i \xi^{T} A k} \\
& =\frac{1}{s} \sum_{i=0}^{s-1} z^{\mu_{i}} \sum_{k \in \mathbb{Z}^{2}} p_{\mu_{i}+A k} z^{A k}=\frac{1}{\sqrt{s}} \sum_{i=0}^{s-1} z^{\mu_{i}} f_{i}^{0}\left(z^{A}\right),
\end{aligned}
$$

where $f_{i}^{0}(x)=(1 / \sqrt{s}) \sum_{k \in \mathbb{Z}^{2}} p_{\mu_{i}+A k} x^{k}, x \in \mathbb{R}^{2}$. Similarly,

$$
m_{l}(\xi)=\frac{1}{\sqrt{s}} \sum_{i=0}^{s-1} z^{\mu_{i}} f_{i}^{l}\left(z^{A}\right), \quad l=1,2, \ldots, N
$$


where $f_{i}^{l}(x)=(1 / \sqrt{s}) \sum_{k \in \mathbb{Z}^{2}} q_{\mu_{i}+A k}^{l} x^{k}, x \in \mathbb{R}^{2}$. Let

$$
\begin{aligned}
& C(z)=\frac{1}{\sqrt{s}} \\
& \times\left(\begin{array}{cccc}
z^{\mu_{0}} e^{-i 2 \pi \mu_{0}^{T} A^{-1} \mu_{0}} & z^{\mu_{1}} e^{-i 2 \pi \mu_{0}^{T} A^{-1} \mu_{1}} & \cdots & z^{\mu_{s-1}} e^{-i 2 \pi \mu_{0}^{T} A^{-1} \mu_{s-1}} \\
z^{\mu_{0}} e^{-i 2 \pi \mu_{1}^{T} A^{-1} \mu_{0}} & z^{\mu_{1}} e^{-i 2 \pi \mu_{1}^{T} A^{-1} \mu_{1}} & \cdots & z^{\mu_{s-1}} e^{-i 2 \pi \mu_{1}^{T} A^{-1} \mu_{s-1}} \\
\vdots & \vdots & & \vdots \\
z^{\mu_{0}} e^{-i 2 \pi \mu_{s-1}^{T} A^{-1} \mu_{0}} & z^{\mu_{1}} e^{-i 2 \pi \mu_{s-1}^{T} A^{-1} \mu_{1}} & \cdots & z^{\mu_{3}} e^{-i 2 \pi \mu_{s-1}^{T} A^{-1} \mu_{s-1}}
\end{array}\right) ;
\end{aligned}
$$

thus,

$$
M(\xi)=C(z)\left(\begin{array}{cccc}
f_{0}^{0}\left(z^{A}\right) & f_{0}^{1}\left(z^{A}\right) & \cdots & f_{0}^{N}\left(z^{A}\right) \\
f_{1}^{0}\left(z^{A}\right) & f_{1}^{1}\left(z^{A}\right) & \cdots & f_{1}^{N}\left(z^{A}\right) \\
\vdots & \vdots & & \vdots \\
f_{s-1}^{0}\left(z^{A}\right) & f_{s-1}^{1}\left(z^{A}\right) & \cdots & f_{s-1}^{N}\left(z^{A}\right)
\end{array}\right)
$$

Since $C(z)$ is a unitary matrix, condition (17) is equivalent to

$$
\begin{aligned}
& \left(\begin{array}{cccc}
f_{0}^{0}\left(z^{A}\right) & f_{0}^{1}\left(z^{A}\right) & \cdots & f_{0}^{N}\left(z^{A}\right) \\
f_{1}^{0}\left(z^{A}\right) & f_{1}^{1}\left(z^{A}\right) & \cdots & f_{1}^{N}\left(z^{A}\right) \\
\vdots & \vdots & & \vdots \\
f_{s-1}^{0}\left(z^{A}\right) & f_{s-1}^{1}\left(z^{A}\right) & \cdots & f_{s-1}^{N}\left(z^{A}\right)
\end{array}\right) \\
& \quad \times\left(\begin{array}{cccc}
f_{0}^{0}\left(z^{A}\right) & f_{0}^{1}\left(z^{A}\right) & \cdots & f_{0}^{N}\left(z^{A}\right) \\
f_{1}^{0}\left(z^{A}\right) & f_{1}^{1}\left(z^{A}\right) & \cdots & f_{1}^{N}\left(z^{A}\right) \\
\vdots & \vdots & & \vdots \\
f_{s-1}^{0}\left(z^{A}\right) & f_{s-1}^{1}\left(z^{A}\right) & \cdots & f_{s-1}^{N}\left(z^{A}\right)
\end{array}\right)=I_{s} .
\end{aligned}
$$

For convenience, let $u=z^{A}$, condition (29) can be rewritten as $\sum_{n=0}^{s-1}\left|f_{n}^{0}(u)\right|^{2} \leq 1$.

If there exists $f_{s}^{0}(u)$ such that

$$
\sum_{n=0}^{s}\left|f_{n}^{0}(u)\right|^{2}=1
$$

then, we have the following Theorem 8 .

Theorem 8. Let $\phi(x) \in L^{2}\left(\mathbb{R}^{2}\right)$ be a compactly supported refinable function, with $\widehat{\phi}$ continuous at 0 and $\widehat{\phi}(0)=1$, and its symbol function satisfies

$$
\sum_{n=0}^{s-1}\left|f_{n}^{0}(u)\right|^{2} \leq 1
$$

If there exists $f_{s}^{0}(u)$ such that

$$
\sum_{n=0}^{s}\left|f_{n}^{0}(u)\right|^{2}=1
$$

then there exists a minimum-energy bivariate wavelet frame associated with $\phi(x)$.
Proof. Under the assumption, we know that the vector

$$
\mathbf{f}:=\left[f_{0}^{0}(u), f_{1}^{0}(u), \ldots, f_{s-1}^{0}(u), f_{s}^{0}(u)\right]^{T}
$$

is a unit vector.

Construct diagonal matrix $D_{0}=\operatorname{diag}\left(u^{t_{0}}, u^{t_{1}}, \ldots, u^{t_{s}}\right)$ such that

$$
\begin{aligned}
\mathbf{f}_{1} & =D_{0} \mathbf{f}=\left[u^{t_{0}} f_{0}^{0}(u), u^{t_{1}} f_{1}^{0}(u), \ldots, u^{t_{s}} f_{s}^{0}(u)\right]^{T} \\
& =\sum_{j=(0,0)}^{K} \mathbf{a}_{j} u^{j}, \quad t_{0}, t_{1}, \ldots, t_{s} \in \mathbb{Z}^{2},
\end{aligned}
$$

where $\mathbf{a}_{j} \in \mathbb{R}^{s+1}$ with $\mathbf{a}_{0} \neq \mathbf{0}$ and $\mathbf{a}_{K} \neq \mathbf{0}$. It is clear that $\mathbf{f}_{1}$ is a unit vector:

$$
\mathbf{f}_{1}^{*} \mathbf{f}_{1}=\left(\sum_{j=(0,0)}^{K} \mathbf{a}_{j} u^{j}\right)^{*}\left(\sum_{j=(0,0)}^{K} \mathbf{a}_{j} u^{j}\right)=1, \quad \forall|u|=1,
$$

and consequently $\mathbf{a}_{0}^{T} \mathbf{a}_{K}=0$. We next consider the $(s+1) \times$ $(s+1)$ Householder matrix:

$$
H_{1}=I_{s+1}-\frac{2}{\|\mathbf{v}\|^{2}} \mathbf{w}^{T},
$$

where $\mathbf{v}=\mathbf{a}_{K} \pm\left\|\mathbf{a}_{K}\right\| \mathbf{e}_{1}$, with $\mathbf{e}_{1}=(1,0, \ldots, 0)_{s+1}^{T}$, and the + or - signs are so chosen that $\mathbf{v} \neq 0$. Then

$$
H_{1} \mathbf{a}_{K}= \pm\left\|\mathbf{a}_{K}\right\| \mathbf{e}_{1} .
$$

Since Householder matrix is orthogonal matrix, we have

$$
\left(H_{1} \mathbf{a}_{0}\right)^{T}\left(H_{1} \mathbf{a}_{K}\right)=\mathbf{a}_{0}^{T} H_{1}^{T} H_{1} \mathbf{a}_{K}=\mathbf{a}_{0}^{T} \mathbf{a}_{K}=0 .
$$

By the previous equation, the first component of $H_{1} \mathbf{a}_{0}$ is 0 . Now $H_{1} \mathbf{f}_{1}=\sum_{j=(0,0)}^{K}\left(H_{1} \mathbf{a}_{j}\right) u^{j}$, we construct diagonal matrix $D_{1}=\operatorname{diag}\left(u^{t_{(1)}}, 1, \ldots, 1\right), t_{(1)} \in \mathbb{Z}^{2}$ such that

$$
\mathbf{f}_{2}=D_{1} H_{1} \mathbf{f}_{1}=D_{1} \sum_{j=(0,0)}^{K}\left(H_{1} \mathbf{a}_{j}\right) u^{j}=\sum_{j=(0,0)}^{K} \mathbf{a}_{j}^{(1)} u^{j}
$$

is also a unit vector and $K_{1}<K, \mathbf{a}_{0}^{(1)} \neq \mathbf{0}, \mathbf{a}_{K_{1}}^{(1)} \neq \mathbf{0}$.

Similarly, we define the Householder matrix:

$$
H_{2}=I_{s+1}-\frac{2}{\|\overline{\mathbf{v}}\|^{2}} \overline{\mathbf{v}} \overline{\mathbf{v}}^{T}
$$

where $\overline{\mathbf{v}}=\mathbf{a}_{K_{1}} \pm\left\|\mathbf{a}_{K_{1}}\right\| \mathbf{e}_{1} \neq \mathbf{0}$, and $D_{2}=\operatorname{diag}\left(u^{t_{(2)}}, 1, \ldots, 1\right)$ such that

$$
\mathbf{f}_{3}=D_{2} H_{2} \mathbf{f}_{2}=D_{2} \sum_{j=(0,0)}^{K_{1}}\left(H_{2} \mathbf{a}_{j}^{(1)}\right) u^{j}=\sum_{j=(0,0)}^{K_{1}} \mathbf{a}_{j}^{(2)} u^{j}
$$

is also a unit vector and $K_{2}<K_{1}, \mathbf{a}_{0}^{(2)} \neq \mathbf{0}, \mathbf{a}_{K_{2}}^{(2)} \neq \mathbf{0}$.

Since every component of $\mathbf{f}$ is a finite sum, we repeat this procedure finite times to get some unitary matrices $D_{L}, H_{L}, D_{L-1}, H_{L-1}, \ldots, H_{2}, D_{1}, H_{1}$,

$$
D_{L} H_{L} D_{L-1} H_{L-1} \cdots H_{2} D_{1} H_{1} D_{0} \mathbf{f}=\mathbf{e}_{1} .
$$


That is, $\mathbf{f}$ is the first column of the unitary matrix

$$
H:=D_{0}^{*} H_{0}^{*} D_{1}^{*} H_{2}^{*} \cdots H_{L-1}^{*} D_{L-1}^{*} H_{L}^{*} D_{L}^{*}
$$

Let

$$
H=\left(\begin{array}{cccc}
f_{0}^{0}(u) & f_{0}^{1}(u) & \cdots & f_{0}^{s}(u) \\
f_{1}^{0}(u) & f_{1}^{1}(u) & \cdots & f_{1}^{s}(u) \\
\vdots & \vdots & & \vdots \\
f_{s}^{0}(u) & f_{s}^{1}(u) & \cdots & f_{s}^{s}(u)
\end{array}\right)
$$

then, $H$ satisfies (38). In the formula (15), we let

$$
m_{l}(\xi)=\frac{1}{\sqrt{s}} \sum_{i=0}^{s-1} z^{\mu_{i}} f_{i}^{l}\left(z^{A}\right), \quad l=1, \ldots, s .
$$

Then,we can obtain the formula (17). The proof of Theorem 8 is completed.

Corollary 9. Let $\phi(x) \in L^{2}\left(\mathbb{R}^{2}\right)$ be a compactly supported refinable function, with $\widehat{\phi}$ continuous at 0 and $\widehat{\phi}(0)=1$, and its symbol function satisfies

$$
\sum_{n=0}^{s-1}\left|m_{0}\left(\xi+2 A^{-1} \mu_{n} \pi\right)\right|^{2} \leq 1, \quad \forall \xi \in \mathbb{R}^{2}
$$

If there exists $f_{s}^{0}(u), \ldots, f_{n}^{0}(u), n \geq s$, such that

$$
\sum_{i=0}^{n}\left|f_{i}^{0}(u)\right|^{2}=1
$$

then there exists a minimum-energy bivariate wavelet frame associated with $\phi(x)$.

Next, we present an explicit formula of constructing minimum-energy bivariate wavelet frame. Suppose that $m_{0}$ satisfies (29); let

$$
\mathscr{D}(\xi)=I_{s}-D(\xi) D^{*}(\xi) \text {. }
$$

Then

$$
\begin{aligned}
& \lambda_{1}=\cdots=\lambda_{s-1}=1, \quad \lambda_{s}=1-\sum_{n=0}^{s-1}\left|m_{0}\left(\xi+2 A^{-T} \mu_{n} \pi\right)\right|^{2} . \\
& \beta_{1}=\frac{1}{\Omega_{1}}\left(m_{0}^{*}\left(\xi+2 A^{-T} \mu_{1} \pi\right),-m_{0}^{*}\left(\xi+2 A^{-T} \mu_{0} \pi\right), 0, \ldots, 0\right)^{T}
\end{aligned}
$$

$$
\begin{aligned}
& \beta_{k}=\frac{1}{\Omega_{k}}\left(-m_{0}\left(\xi+2 A^{-T} \mu_{0} \pi\right) m_{0}^{*}\left(\xi+2 A^{-T} \mu_{k} \pi\right),\right. \\
& -m_{0}\left(\xi+2 A^{-T} \mu_{1} \pi\right) m_{0}^{*}\left(\xi+2 A^{-T} \mu_{k} \pi\right), \ldots, \\
& -m_{0}\left(\xi+2 A^{-T} \mu_{k-1} \pi\right) m_{0}^{*}\left(\xi+2 A^{-T} \mu_{k} \pi\right), \\
& \left.\sum_{j=0}^{k-1}\left|m_{0}\left(\xi+2 A^{-T} \mu_{j} \pi\right)\right|^{2}, 0, \ldots, 0\right)^{T} \\
& k=2,3, \ldots, s-1 \text {, } \\
& \beta_{s}=\frac{1}{\Omega_{s}}\left(m_{0}\left(\xi+2 A^{-T} \mu_{0} \pi\right),\right. \\
& \left.m_{0}\left(\xi+2 A^{-T} \mu_{1} \pi\right), \ldots, m_{0}\left(\xi+2 A^{-T} \mu_{s-1} \pi\right)\right)^{T}
\end{aligned}
$$

are the eigenvalues and eigenvectors of $\mathscr{D}(\xi)$, respectively, where $\Omega_{1}, \Omega_{2}, \ldots, \Omega_{s}$ ensure that $\beta_{1}, \beta_{2}, \ldots, \beta_{s}$ are unit vector. Let

$$
S(\xi)=\left(\begin{array}{llll}
\beta_{1} & \beta_{2} & \cdots & \beta_{s}
\end{array}\right), \quad \Lambda(\xi)=\operatorname{diag}\left(1, \ldots, 1, \lambda_{s}\right),
$$

then,

$$
I_{s}-D(\xi) D^{*}(\xi)=S(\xi) \Lambda(\xi) S^{*}(\xi) .
$$

Theorem 10. Let $\phi(x) \in L^{2}\left(\mathbb{R}^{2}\right)$ be a compactly supported refinable function, with $\widehat{\phi}$ continuous at 0 and $\widehat{\phi}(0)=1$, and its symbol function satisfies

$$
\sum_{n=0}^{s-1}\left|m_{0}\left(\xi+2 A^{-1} \mu_{n} \pi\right)\right|^{2} \leq 1, \quad \forall \xi \in \mathbb{R}^{2} .
$$

If there exists $f_{s}^{0}(u)$ satisfies

$$
\sum_{i=0}^{s}\left|f_{i}^{0}(u)\right|^{2}=1
$$

then the symbol function of minimum-energy bivariate wavelet frame associated with $\phi(x)$ is the first row of the matrix:

$$
Q(\xi)=U(\xi) \sqrt{\Lambda(\xi)} S(\xi),
$$

where $U(\xi)$ is any $s \times s$ unitary matrix, and $S(\xi), \Lambda(\xi)$ are defined in (59).

Proof. By Theorem 8, there exists a minimum-energy bivariate wavelet frame associated with the given scaling function, and the existence of $f_{s}^{0}(u)$ guarantees that the elements of $\sqrt{\Lambda(\xi)}$ are trigonometric polynomial, and we have

$$
\begin{aligned}
Q(\xi) Q^{*}(\xi) & =S(\xi) \sqrt{\Lambda(\xi)} U(\xi)(S(\xi) \sqrt{\Lambda(\xi)} U(\xi))^{*} \\
& =S(\xi) \Lambda(\xi) S^{*}(\xi)=I_{s}-D(\xi) D^{*}(\xi) ;
\end{aligned}
$$

thus, symbol functions satisfy (17).

Theorems 8 and 10 give different methods to construct minimum-energy bivariate wavelet frame. 


\section{Decomposition and Reconstruction Formulas of Minimum-Energy Bivariate Wavelet Frames}

Suppose that the bivariate scaling function $\phi(x)$ has an associated minimum-energy bivariate wavelet frame $\left\{\psi^{1}, \ldots, \psi^{N}\right\}$. Let the projection operators $\mathbf{P}_{j}$ of $L^{2}\left(\mathbb{R}^{2}\right)$ onto the nested subspace $V_{j}$ be defined by

$$
\mathbf{P}_{j} f:=\sum_{k \in \mathbb{Z}^{2}}\left\langle f, \phi_{j, k}\right\rangle \phi_{j, k}
$$

Then the formula (12) can be rewritten as

$$
\mathbf{P}_{j+1} f-\mathbf{P}_{j} f:=\sum_{i=1}^{N} \sum_{k \in \mathbb{Z}^{2}}\left\langle f, \psi_{j, k}^{i}\right\rangle \psi_{j, k}^{i} .
$$

In other words, the error term $g_{j}=\mathbf{P}_{j+1} f-\mathbf{P}_{j} f$ between consecutive projections is given by the frame expansion:

$$
g_{j}=\sum_{i=1}^{N} \sum_{k \in \mathbb{Z}^{2}}\left\langle f, \psi_{j, k}^{i}\right\rangle \psi_{j, k}^{i}
$$

Suppose that the error term $g_{j}$ has another expansion in terms of the frames $\left\{\psi^{1}, \ldots, \psi^{N}\right\}$, that is,

$$
g_{j}=\sum_{i=1}^{N} \sum_{k \in \mathbb{Z}^{2}} c_{j, k} \psi_{j, k}^{i}
$$

Then by using both (67) and (68), we have

$$
\left\langle g_{j}, f\right\rangle=\sum_{i=1}^{N} \sum_{k \in \mathbb{Z}^{2}}\left|\left\langle f, \psi_{j, k}^{i}\right\rangle\right|^{2}=\sum_{i=1}^{N} \sum_{k \in \mathbb{Z}^{2}} c_{j, k} \overline{\left\langle f, \psi_{j, k}^{i}\right\rangle},
$$

and this derives

$$
\begin{aligned}
0 \leq & \sum_{i=1}^{N} \sum_{k \in \mathbb{Z}^{2}}\left|c_{j, k}-\left\langle f, \psi_{j, k}^{i}\right\rangle\right|^{2} \\
= & \sum_{i=1}^{N} \sum_{k \in \mathbb{Z}^{2}}\left|c_{j, k}\right|^{2}-2 \sum_{i=1}^{N} \sum_{k \in \mathbb{Z}^{2}} c_{j, k} \overline{\left\langle f, \psi_{j, k}^{i}\right\rangle} \\
& +\sum_{i=1}^{N} \sum_{k \in \mathbb{Z}^{2}}\left|\left\langle f, \psi_{j, k}^{i}\right\rangle\right|^{2} \\
= & \sum_{i=1}^{N} \sum_{k \in \mathbb{Z}^{2}}\left|c_{j, k}\right|^{2}-\sum_{i=1}^{N} \sum_{k \in \mathbb{Z}^{2}}\left|\left\langle f, \psi_{j, k}^{i}\right\rangle\right|^{2} .
\end{aligned}
$$

This inequality means that the coefficients of the error term $g_{j}$ in (67) have minimal $l^{2}$-norm among all sequences $\left\{c_{j, k}\right\}$ which satisfy (68).

We next discuss minimum-energy bivariate wavelet frames decomposition and reconstruction. For any $f \in$ $L^{2}\left(\mathbb{R}^{2}\right)$, we define the coefficients

$$
c_{j, k}:=\left\langle f, \phi_{j, k}\right\rangle, \quad d_{j, k}:=\left\langle f, \psi_{j, k}^{i}\right\rangle \quad i=1, \ldots, N .
$$

Then we can derive the decomposition and reconstruction formulas that are similar to those of orthonormal wavelets.

(1) Decomposition Algorithm. Suppose that coefficients $\left\{c_{j+1, l}\right.$ : $\left.l \in \mathbb{Z}^{2}\right\}$ are known. By the two-scale relations (8) and (13), we have

$$
\begin{gathered}
\phi_{j, l}(x)=\frac{1}{\sqrt{s}} \sum_{k \in \mathbb{Z}^{2}} p_{k-A l} \phi_{j+1, k}(x), \\
\psi_{j, l}^{i}(x)=\frac{1}{\sqrt{s}} \sum_{k \in \mathbb{Z}^{2}} q_{k-A l}^{i} \psi_{j+1, k}^{i}(x), \quad i=1, \ldots, N .
\end{gathered}
$$

Then, decomposition algorithm is given as

$$
\begin{gathered}
c_{j, l}=\frac{1}{\sqrt{s}} \sum_{k \in \mathbb{Z}^{2}} p_{k-A l} c_{j+1, k}, \\
d_{j, l}^{i}=\frac{1}{\sqrt{s}} \sum_{k \in \mathbb{Z}^{2}} q_{k-A l}^{i} d_{j+1, k}^{i}, \quad i=1, \ldots, N .
\end{gathered}
$$

(2) Reconstruction Algorithm. From (26), it follows that

$$
\phi_{j+1, l}(x)=\frac{1}{\sqrt{s}} \sum_{k \in \mathbb{Z}^{2}}\left\{p_{l-A k}^{*} \phi_{j, k}(x)+\sum_{i=1}^{N} q_{l-A k}^{i *} \psi_{j, k}^{i}(x)\right\} .
$$

Take the inner products on both sides of (74) with $f$, we get

$$
c_{j+1, l}=\frac{1}{\sqrt{s}} \sum_{k \in \mathbb{Z}^{2}}\left\{p_{l-A k}^{*} c_{j, k}+\sum_{i=1}^{N} q_{l-A k}^{i *} d_{j, k}^{i}\right\} .
$$

\section{Numerical Examples}

In this section, we present some numerical examples to show the effectiveness of the proposed methods.

Example 1. Let $A=2 I_{2}$; then $s=4$ and

$$
\begin{array}{ll}
\mu_{0}=(0,0)^{T}, & \mu_{1}=(1,0)^{T}, \\
\mu_{2}=(0,1)^{T}, & \mu_{3}=(1,1)^{T} .
\end{array}
$$

Suppose that the Fourier transform $\widehat{\phi}(\xi)$ of scaling function $\phi(\xi)$ is

$$
\widehat{\phi}(\xi)=\frac{1-e^{-i \xi_{1}}}{i \xi_{1}} \cdot \frac{1-e^{-i \xi_{2}}}{i \xi_{2}} \cdot \frac{1-e^{-i\left(\xi_{1}+\xi_{2}\right)}}{i\left(\xi_{1}+\xi_{2}\right)} .
$$

Then its symbol function $m_{0}(\xi)$ satisfies

$$
m_{0}(\xi)=\frac{1}{8}\left(1+e^{-i \xi_{1}}\right)\left(1+e^{-i \xi_{2}}\right)\left(1+e^{-i\left(\xi_{1}+\xi_{2}\right)}\right) .
$$

Thus,

$$
\begin{gathered}
f_{0}^{0}(u)=\frac{1}{4}\left(1+u^{(1,1)^{T}}\right), \quad f_{1}^{0}(u)=\frac{1}{4}\left(1+u^{(0,1)^{T}}\right), \\
f_{2}^{0}(u)=\frac{1}{4}\left(1+u^{(1,0)^{T}}\right), \quad f_{3}^{0}(u)=\frac{2}{4} .
\end{gathered}
$$


Let

$$
\begin{gathered}
f_{4}^{0}(u)=\frac{1}{4}\left(1-u^{(1,0)^{T}}\right), \quad f_{5}^{0}(u)=\frac{1}{4}\left(1-u^{(0,1)^{T}}\right), \\
f_{6}^{0}(u)=\frac{1}{4}\left(1-u^{(1,1)^{T}}\right) .
\end{gathered}
$$

Then we have $\sum_{l=0}^{6}\left|f_{i}^{0}(u)\right|^{2}=1$. By Corollary 9 , there exists a minimum-energy bivariate wavelet frame associated with $\phi(\xi)$. Using Theorem 8 , let

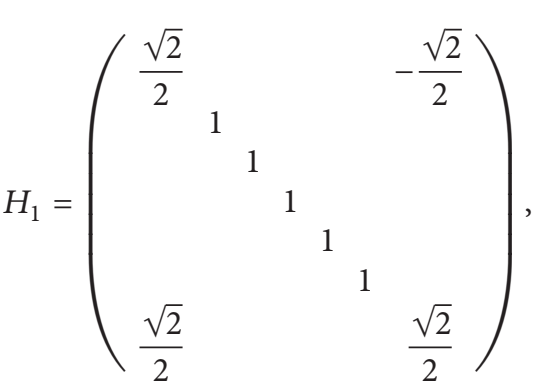

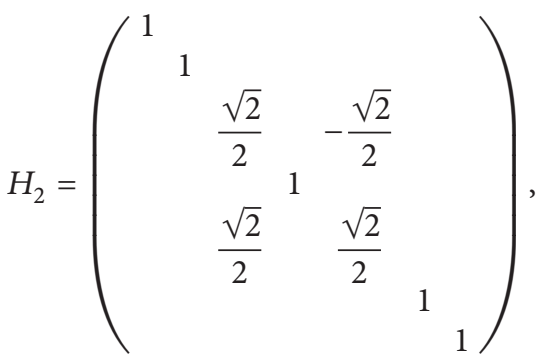

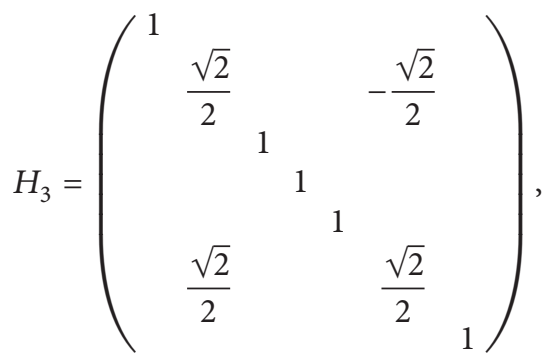

$\mathrm{H}_{4}$

$$
\begin{gathered}
=\frac{1}{4}\left(\begin{array}{ccccccc}
\sqrt{2} & \sqrt{2} & \sqrt{2} & 2 & \sqrt{2} & \sqrt{2} & \sqrt{2} \\
0 & 0 & 2 & -2 \sqrt{2} & 2 & 0 & 0 \\
2 \sqrt{2} & -2 \sqrt{2} & 0 & 0 & 0 & 0 & 0 \\
0 & 0 & 0 & 0 & 0 & -2 \sqrt{2} & 2 \sqrt{2} \\
0 & 0 & 2 \sqrt{2} & 0 & -2 \sqrt{2} & 0 & 0 \\
2 & 2 & 0 & 0 & 0 & -2 & -2 \\
\sqrt{2} & \sqrt{2} & -\sqrt{2} & -2 & -\sqrt{2} & \sqrt{2} & \sqrt{2}
\end{array}\right), \\
D_{1}=\operatorname{diag}\left(u^{(-1,-1)}, 1,1,1,1,1,1\right) \\
D_{2}=\operatorname{diag}\left(1,1, u^{(-1,0)}, 1,1,1,1\right) \\
D_{3}=\operatorname{diag}\left(1, u^{(0,-1)}, 1,1,1,1,1\right) .
\end{gathered}
$$

Thus, we have

$$
H_{1}^{*} D_{1}^{*} H_{2}^{*} D_{2}^{*} H_{3}^{*} D_{3}^{*} H_{4}^{*}=\frac{1}{4}\left(\begin{array}{ccccccc}
1+u^{(1,1)} & 0 & 2 u^{(1,1)} & 2 & 0 & -\sqrt{2}+\sqrt{2} u^{(1,1)} & 1+u^{(1,1)} \\
1+u^{(0,1)} & 0 & -2 u^{(0,1)} & -2 & 0 & -\sqrt{2}+\sqrt{2} u^{(0,1)} & 1+u^{(0,1)} \\
1+u^{(1,0)} & \sqrt{2}+\sqrt{2} u^{(1,0)} & 0 & 0 & -2+2 u^{(1,0)} & 0 & -1-u^{(1,0)} \\
2 & -2 \sqrt{2} & 0 & 0 & 0 & 0 & -2 \\
1-u^{(1,0)} & \sqrt{2}-\sqrt{2} u^{(1,0)} & 0 & 0 & -2-2 u^{(1,0)} & 0 & -1+u^{(1,0)} \\
1-u^{(0,1)} & 0 & 2 u^{(0,1)} & -2 & 0 & -\sqrt{2}-\sqrt{2} u^{(0,1)} & 1-u^{(0,1)} \\
1-u^{(1,1)} & 0 & -2 u^{(1,1)} & 2 & 0 & -\sqrt{2}-\sqrt{2} u^{(1,1)} & 1-u^{(1,1)}
\end{array}\right) .
$$

Consequently, we obtain symbol functions

$$
\left.-e^{-i \xi_{2}}\left(1+e^{-i 2 \xi_{1}}\right)-2 e^{-i\left(\xi_{1}+\xi_{2}\right)}\right)
$$

$$
\begin{aligned}
& m_{1}(\xi)=\frac{1}{8}\left(e^{-i \xi_{2}}\left(\sqrt{2}+\sqrt{2} e^{-i 2 \xi_{1}}\right)-2 \sqrt{2} e^{-i\left(\xi_{1}+\xi_{2}\right)}\right) \\
& m_{2}(\xi)=\frac{1}{8}\left(2 e^{-i 2\left(\xi_{1}+\xi_{2}\right)}-2 e^{-i \xi_{1}} e^{-i 2 \xi_{1}}\right) \\
& m_{3}(\xi)=\frac{1}{8}\left(2-2 e^{-i \xi_{1}}\right) \\
& m_{4}(\xi)=\frac{1}{8} e^{-i \xi_{2}}\left(-2+2 e^{-i 2 \xi_{1}}\right) \\
& m_{5}(\xi)=\frac{1}{8}\left(-\sqrt{2}+\sqrt{2} e^{-i\left(2 \xi_{1}+2 \xi_{2}\right)}+e^{-i \xi_{1}}\left(-\sqrt{2}+\sqrt{2} e^{-i 2 \xi_{2}}\right)\right) \\
& m_{6}(\xi)=\frac{1}{8}\left(1+e^{-i\left(2 \xi_{1}+2 \xi_{2}\right)}+e^{-i \xi_{1}}\left(1+e^{-i 2 \xi_{2}}\right)\right.
\end{aligned}
$$

The functions generated by the previous symbol functions are minimum-energy bivariate wavelet frame functions associated with $\phi(x)$.

Example 2. Let $A=3 I_{2}$; then $s=9$ and

$$
\begin{array}{lll}
\mu_{0}=(0,0)^{T}, & \mu_{1}=(1,0)^{T}, & \mu_{2}=(2,0)^{T}, \\
\mu_{3}=(0,1)^{T}, & \mu_{4}=(0,2)^{T}, & \mu_{5}=(1,1)^{T}, \\
\mu_{6}=(2,1)^{T}, & \mu_{7}=(1,2)^{T}, & \mu_{8}=(2,2)^{T} .
\end{array}
$$


Suppose that the Fourier transform $\widehat{\phi}(\xi)$ of scaling function $\phi(\xi)$ is

$$
\widehat{\phi}(\xi)=\frac{1-e^{-i \xi_{1}}}{i \xi_{1}} \cdot \frac{1-e^{-i \xi_{2}}}{i \xi_{2}} \cdot \frac{1-e^{-i\left(\xi_{1}+\xi_{2}\right)}}{i\left(\xi_{1}+\xi_{2}\right)} .
$$

Then its symbol function $m_{0}(\xi)$ satisfies

$$
\begin{aligned}
m_{0}(\xi)= & \frac{1}{27}\left(1+e^{-i \xi_{1}}+e^{-i 2 \xi_{1}}\right)\left(1+e^{-i \xi_{2}}+e^{-i 2 \xi_{2}}\right) \\
& \times\left(1+e^{-i\left(\xi_{1}+\xi_{2}\right)}+e^{-i 2\left(\xi_{1}+\xi_{2}\right)}\right) .
\end{aligned}
$$

Thus,

$$
\begin{aligned}
& f_{0}^{0}(u)=\frac{1}{9}\left(1+u^{(1,1)^{T}}\right), \\
& f_{1}^{0}(u)=\frac{1}{9}\left(1+u^{(0,1)^{T}}+u^{(1,1)^{T}}\right), \\
& f_{2}^{0}(u)=\frac{1}{9}\left(1+2 u^{(0,1)^{T}}\right), \\
& f_{3}^{0}(u)=\frac{1}{9}\left(1+u^{(1,0)^{T}}+u^{(1,1)^{T}}\right), \\
& f_{4}^{0}(u)=\frac{1}{9}\left(1+2 u^{(1,0)^{T}}\right), \quad f_{5}^{0}(u)=\frac{1}{9}\left(2+u^{(1,1)^{T}}\right), \\
& f_{6}^{0}(u)=\frac{1}{9}\left(2+u^{(0,1)^{T}}\right), \quad f_{7}^{0}(u)=\frac{1}{9}\left(2+u^{(1,0)^{T}}\right), \\
& f_{8}^{0}(u)=\frac{3}{9} .
\end{aligned}
$$

Let

$$
\begin{gathered}
f_{9}^{0}(u)=\frac{\sqrt{6}}{9}\left(1-u^{(1,0)^{T}}\right), \quad f_{10}^{0}(u)=\frac{\sqrt{6}}{9}\left(1-u^{(0,1)^{T}}\right), \\
f_{11}^{0}(u)=\frac{\sqrt{6}}{9}\left(1-u^{(1,1)^{T}}\right) .
\end{gathered}
$$

Then we have $\sum_{l=0}^{11}\left|f_{l}^{0}(u)\right|^{2}=1$. By Corollary 9 , there exists a minimum-energy bivariate wavelet frame associated with $\phi(\xi)$.

Example 3. Let $A=\left(\begin{array}{cc}1 & 1 \\ 1 & -1\end{array}\right)$; then $s=2$ and

$$
\mu_{0}=(0,0)^{T}, \quad \mu_{1}=(1,0)^{T} .
$$

Suppose that the Fourier transform $\widehat{\phi}(\xi)$ of scaling function $\phi(\xi)$ satisfies

$$
\widehat{\phi}(\xi)=\frac{1-e^{-i \xi_{1}}}{i \xi_{1}} \cdot \frac{1-e^{-i \xi_{2}}}{i \xi_{2}} \cdot \frac{1-e^{-i\left(\xi_{1}+\xi_{2}\right)}}{i\left(\xi_{1}+\xi_{2}\right)} \cdot \frac{1-e^{-i\left(\xi_{1}-\xi_{2}\right)}}{i\left(\xi_{1}-\xi_{2}\right)} .
$$

Then its symbol function $m_{0}(\xi)$ satisfies

$$
m_{0}(\xi)=\frac{1}{4}\left(1+e^{-i \xi_{1}}\right)\left(1+e^{-i \xi_{2}}\right) .
$$

Let $z=e^{-i \xi^{T}}=\left(e^{-i \xi_{1}} e^{-i \xi_{2}}\right)^{T}$, and we have $z^{\mu_{0}}=1, z^{\mu_{1}}=$ $e^{-i \xi_{1}}, u=z^{A}=\left(e^{-i\left(\xi_{1}+\xi_{2}\right)}, e^{-i\left(\xi_{1}-\xi_{2}\right)}\right)$. Therefore,

$$
f_{0}^{0}(u)=\frac{1}{2 \sqrt{2}}\left(1+u^{(1,0)^{T}}\right), \quad f_{1}^{0}(u)=\frac{1}{2 \sqrt{2}}\left(1+u^{(0,-1)^{T}}\right) .
$$

Let

$f_{2}^{0}(u)=\frac{1}{2 \sqrt{2}}\left(1-u^{(1,0)^{T}}\right), \quad f_{3}^{0}(u)=\frac{1}{2 \sqrt{2}}\left(1-u^{(0,-1)^{T}}\right)$,

and we have $\sum_{l=0}^{3}\left|f_{l}^{0}(u)\right|^{2}=1$. By Corollary 9 , there exists a minimum-energy bivariate wavelet frame associated with $\phi(\xi)$.

\section{Conclusions}

In this paper, we define the concept of minimum-energy bivariate wavelet frame with arbitrary dilation matrix and present its equivalent characterizations. We give a necessary condition and two sufficient conditions for minimum-energy bivariate wavelets frame and deduce the decomposition and reconstruction formulas of minimum-energy bivariate wavelets frame. Finally, we give several numerical examples to show the effectiveness of the proposed methods.

\section{Acknowledgments}

The authors would like to thank Professor Weili Li and the anonymous reviewers for suggesting many helpful improvements to this paper. This work was supported by the National Natural Science Foundation of China under the Project Grant no. 61261043, Natural Science Foundation of Ningxia under the Project the Grant no. NX13xxx, and the Research Project of Beifang University of Nationalities under the Project no. 2012Y036.

\section{References}

[1] R. J. Duffin and A. C. Schaeffer, "A class of nonharmonic Fourier series," Transactions of the American Mathematical Society, vol. 72, pp. 341-366, 1952.

[2] I. Daubechies, A. Grossmann, and Y. Meyer, "Painless nonorthogonal expansions," Journal of Mathematical Physics, vol. 27, no. 5, pp. 1271-1283, 1986.

[3] J. J. Benedetto and S. Li, "The theory of multiresolution analysis frames and applications to filter banks," Applied and Computational Harmonic Analysis, vol. 5, no. 4, pp. 389-427, 1998.

[4] C. K. Chui and X. L. Shi, "Orthonormal wavelets and tight frames with arbitrary real dilations," Applied and Computational Harmonic Analysis, vol. 9, no. 3, pp. 243-264, 2000.

[5] C. K. Chui and W. He, "Compactly supported tight frames associated with refinable functions," Applied and Computational Harmonic Analysis, vol. 8, no. 3, pp. 293-319, 2000.

[6] A. Petukhov, "Symmetric framelets," Constructive Approximation, vol. 19, no. 2, pp. 309-328, 2003. 
[7] Y. Huang and Z. Cheng, "Minimum-energy frames associated with refinable function of arbitrary integer dilation factor," Chaos, Solitons \& Fractals, vol. 32, no. 2, pp. 503-515, 2007.

[8] X. Gao and C. H. Cao, "Minimum-energy wavelet frame on the interval," Science in China F, vol. 51, no. 10, pp. 1547-1562, 2008.

[9] Q. Liang and P. Zhao, "Minimum-energy multi-wavelets tight frames associatedWith two scaling functions," in Proceedings of the International Conference on Wavelet Analysis and Pattern Recognition, vol. 1, pp. 97-101, 2010.

[10] Y. D. Huang, Q. F. Li, and M. Li, "Minimum-energy multiwavelet frames on the interval with dilation factor," Mathematical Problem in Engineering, vol. 2012, Article ID 640789, 37 pages, 2012.

[11] Y. D. Huang and Q. F. Li, "Minimum-energy wavelet frame on the interval with arbitrary dilation factor," Science China Information Science, vol. 43, no. 4, pp. 469-487, 2013.

[12] M. J. Lai and J. Stöckler, "Construction of multivariate compactly supported tight wavelet frames," Applied and Computational Harmonic Analysis, vol. 21, no. 3, pp. 324-348, 2006.

[13] J. F. Cheng and D. F. Li, "Characterization of MRA E-tight frame wavelet," Acta Mathematica Sinica (Chinese Series), vol. 51, no. 5, pp. 877-888, 2008.

[14] Q. F. Lian and Y. Z. Li, "A class of higher-dimensional FMRA wavelet frames," Acta Mathematica Sinica (Chinese Series), vol. 52, no. 5, pp. 853-860, 2009.

[15] F. Y. Zhou and Y. Z. Li, "Multivariate FMRAs and FMRA frame wavelets for reducing subspaces of $L^{2}\left(\mathbb{R}^{d}\right)$," Kyoto Journal of Mathematics, vol. 50, no. 1, pp. 83-99, 2010.

[16] Y. D. Huang and N. Sun, "Characterizations of A-Parseval frame wavelet," Acta Mathematica Sinica (Chinese Series), vol. 54, no. 5, pp. 767-790, 2011.

[17] Y. Zhang and T. Q. Lv, "Study of tight bivariate wavelet frames with multiscale and application in information science," Advances in Intelligent and Soft Computing, vol. 159, pp. 125-131, 2012.

[18] O. Christensen, An Introduction to Frames and Riesz Bases, Applied and Numerical Harmonic Analysis, Birkhäauser, Boston, Mass, USA, 2003. 


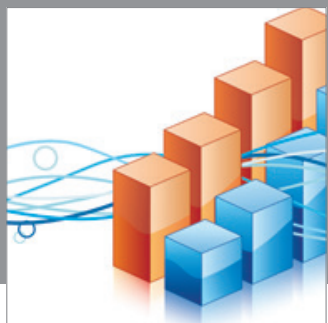

Advances in

Operations Research

mansans

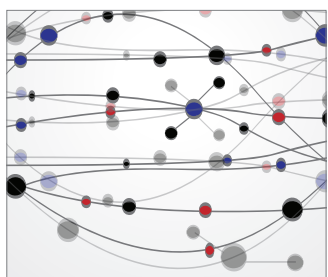

The Scientific World Journal
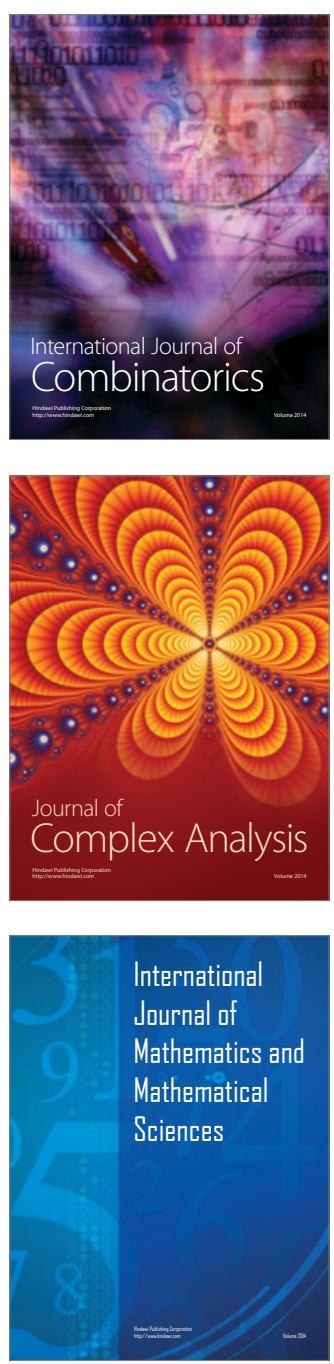
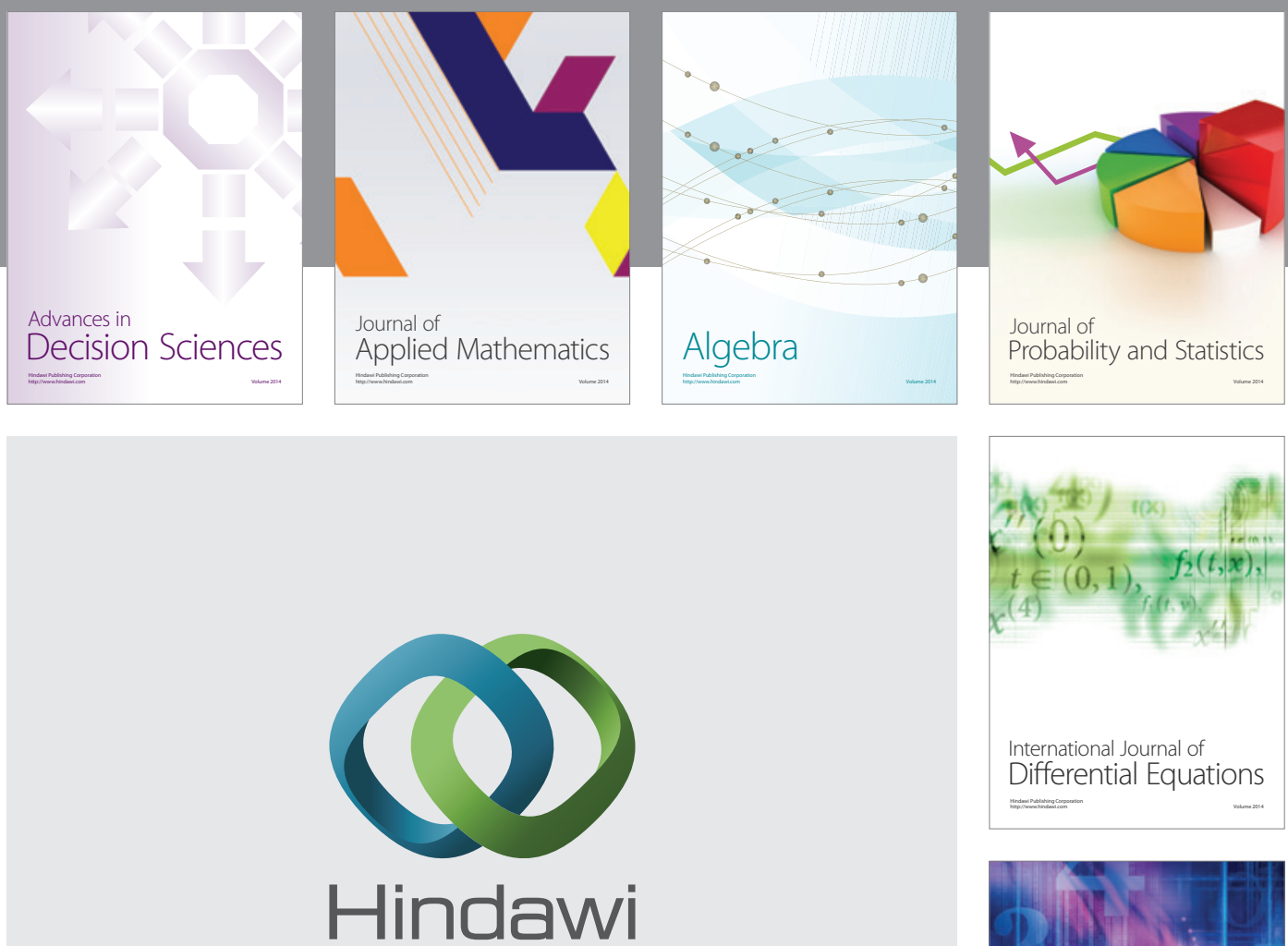

Submit your manuscripts at http://www.hindawi.com
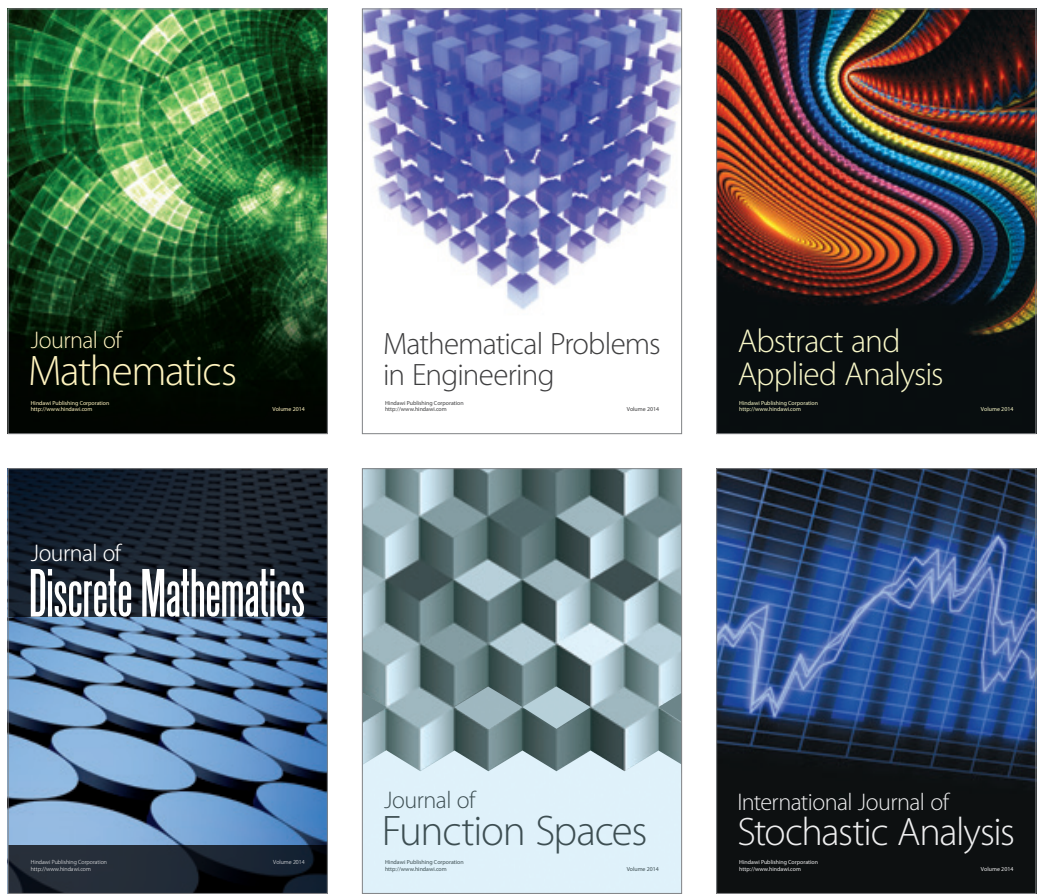

Journal of

Function Spaces

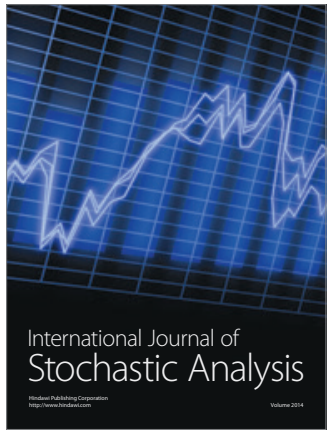

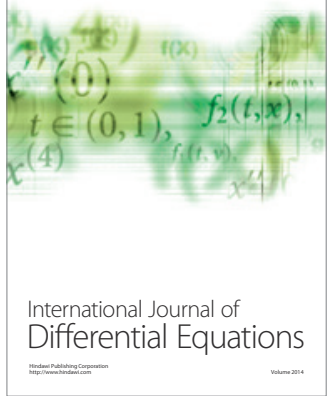
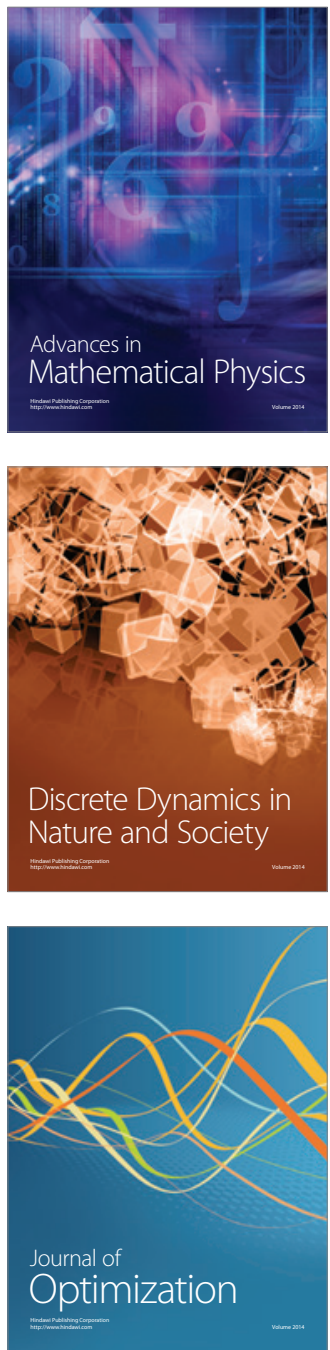